\title{
Law of universal gravitation with finite velocity of gravity and mathematical model of motion of a finite number of material points
}

https://doi.org/10.31713/MCIT.2020.25

\author{
V. Yu. Slyusarchuk \\ Department of Higher Mathematics \\ National University of Water and Enviromental Engineering \\ Rivne, Ukraine \\ e-mail: V.E.Slyusarchuk@gmail.com
}

\begin{abstract}
The law of universal gravitation is introduced taking into account the finiteness of the gravitational velocity. Based on this law, a mathematical

model of the motion of a finite number of material points is constructed, a separate case of which is the classical model of the motion of points, which is described by a system of ordinary differential equations. The constructed model is a system of nonlinear differential equations with deviating argument and functional equations. It more accurately describes the dynamics of a finite number of material points than the corresponding classical model. A mathematical model of the motion of two material points is also considered.
\end{abstract}

Keywords: law of universal gravitation with finite velocity of gravity, mathematical model of motion of a finite number of material points.

\section{INTRODUCTION}

In Newton's theory of gravity, the gravity velocity $c_{g}$ is not included in any formula and is considered to be infinitely large $\left(c_{g}=\infty\right)$.

In A. Einstein's general theory of relativity for the speed of gravity, it is assumed that $c_{g}=c$, where $c$ is the speed of light [1], [2].

The speed of gravity can be estimated by the transmission rate of the influence of the gravitational field on the measurement results. So, in 2002, Kopeikin and Fomalont conducted an experiment based on radio interferometry with an extremely long base, in which radiation from a distant quasar QSO J0842+1835, which passed near a massive body - Jupiter, was recorded by Earth's radio telescopes [3]. Analysis of the experimental data yielded a speed of gravity close in magnitude to the speed of light, with an accuracy of about $20 \%$.

The second method of measuring the speed of gravity is associated with the fixation of gravitational waves from distant stellar sources simultaneously with a light signal.
The first such measurement was obtained in 2017 for the GW170817 gravitational wave generated by the fusion of two neutron stars (the distance to the source was $13 \times 10^{7}$ light years) using laser-interferometric gravitational-wave detectors of the LIGO-Virgo detector grid. Behind this event, the deviation of the speed of gravitational waves from the speed of light, if such a deviation exists, ranges from $-3 \times 10^{-15}$ to $+0.7 \times 10^{-15}$, those are compatible with zero within the error [4].

This article is devoted to the law of universal gravitation, taking into account the finiteness of the speed of gravity and its application to the construction of a mathematical model of motion of a finite number of bodies. Such a model is not a system of ordinary differential equations, as in classical celestial mechanics [5], but a system of differential equations with a deviating argument and functional equations, as in [6]. The dynamics of bodies, taking into account the finiteness of the gravity velocity, is characterized by properties that are impossible in the case of $c_{g}=\infty$.

\section{THE LAW OF GRAVITY WITH FINITE SPEED OF GRAVITY}

Consider two material points $M_{1}$ and $M_{2}$ with masses $m_{1}$ and $m_{2}$ respectively. According to the law of universal gravitation and Newton's second law, these points move in space. The movement of the points will be considered with respect to the rectangular coordinate system $x, y, z$ with the origin at some point $O$. We consider that the coordinate system is inertial and only gravity generated by another point acts on each point. The position of the points $M_{1}$ and $M_{2}$ at time $t$ is determined by their vector functions $\vec{r}_{1}(t)$ and $\vec{r}_{2}(t)$.

To study the motion of points $M_{1}$ and $M_{2}$, it is necessary to know the forces with which each of these points attracts the other.

If the speed of gravity were infinite, as in Newton's theory, then, on the basis of the law of universal gravitation, 


\section{Modeling, control and information technologies - 2020}

at the moment $t$, the point $M_{2}$ would attract the point $M_{1}$ with force

$$
\vec{F}_{2,1, \infty}(t)=\frac{G m_{1} m_{2}}{\left|\vec{r}_{2}(t)-\vec{r}_{1}(t)\right|^{3}}\left(\vec{r}_{2}(t)-\vec{r}_{1}(t)\right),
$$

where $G$ is the gravitational constant and $\left|\vec{r}_{2}(t)-\vec{r}_{1}(t)\right|$ is the Euclidean length of the vector $\vec{r}_{2}(t)-\vec{r}_{1}(t)$. The direction of this force coincides with the direction of vector $\vec{r}_{2}(t)-\vec{r}_{1}(t)$. force

Similarly, at time $t$, point $M_{1}$ attracted point $M_{2}$ with

$$
\vec{F}_{1,2, \infty}(t)=\frac{G m_{1} m_{2}}{\left|\vec{r}_{1}(t)-\vec{r}_{2}(t)\right|^{3}}\left(\vec{r}_{1}(t)-\vec{r}_{2}(t)\right) .
$$

According to paragraph 1 , the speed of gravity is finite. Therefore, the action of one point on another is carried out taking into account the delay of the gravitational field.

Let us explain the effect of the delay of the gravitational field on the forces with which the considered points 1 and 2 are attracted.

Based on the finiteness of the speed of gravity, a another force acts on point $M_{1}$

$$
\begin{gathered}
\vec{F}_{2,1, c}(t)= \\
=\frac{G m_{1} m_{2}}{\left|\vec{r}_{2}\left(t-\tau_{2,1}(t)\right)-\vec{r}_{1}(t)\right|^{3}}\left(\vec{r}_{2}\left(t-\tau_{2,1}(t)\right)-\vec{r}_{1}(t)\right),
\end{gathered}
$$

where the delay of gravity $\tau_{2,1}(t)$ in (2) satisfies the ratio of

$$
c \tau_{2,1}(t)=\left|\vec{r}_{2}\left(t-\tau_{2,1}(t)\right)-\vec{r}_{1}(t)\right|
$$

and $c$ the speed of gravity.

Indeed, let points $M_{2}$ and $M_{1}$ move along curves, parts of which are shown in Fig. 1, with speeds

$$
\vec{v}_{2}(t)=\frac{d \vec{r}_{2}(t)}{d t} \text { and } \vec{v}_{1}(t)=\frac{d \vec{r}_{1}(t)}{d t} .
$$

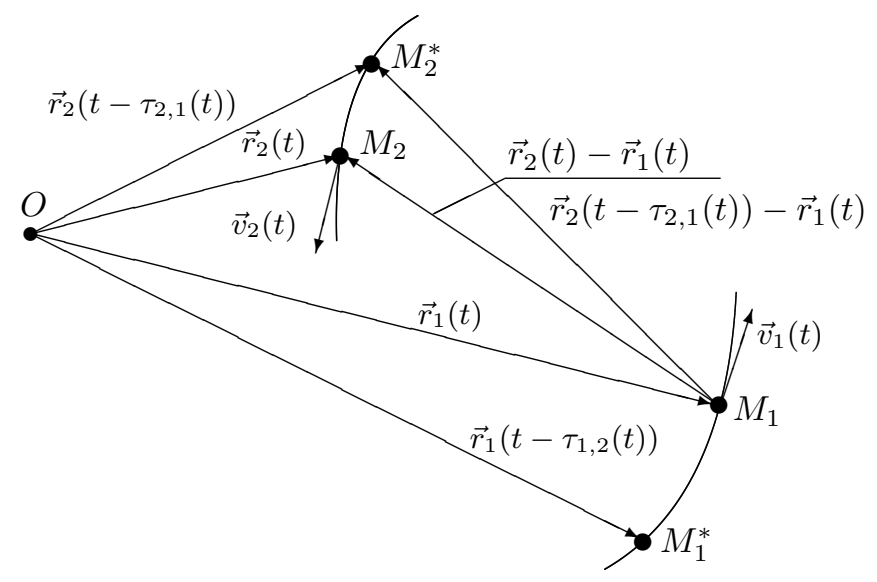

Fig. 1. Points $M_{1}$ and $M_{2}$ at times $t$ and $t-\tau_{2,1}(t)$.

Let point $M_{2}$ at time $t-\tau_{2,1}(t)$, where $\tau_{2,1}(t)$ satisfies
(3), coincide with point $M_{2}^{*}$. Time interval $\left[t-\tau_{2,1}(t), t\right]$ corresponds to curve $M_{2}^{*} M_{2}$, along which point $M_{2}$ moves. This time interval is sufficient for the gravitational field with a velocity of $c$ to propagate from the point $M_{2}^{*}$ to the point $M_{1}$. Thus, at time $t$, point $M_{1}$ is not affected by force (1), but by force (2).

Note that the attracting point for point $M_{1}$ at time $t$ is not point $M_{2}$, but point $M_{2}^{*}$.

Similarly, based on the finiteness of the speed of gravity, force

$$
\begin{gathered}
\vec{F}_{1,2, c}(t)= \\
=\frac{G m_{1} m_{2}}{\left|\vec{r}_{1}\left(t-\tau_{1,2}(t)\right)-\vec{r}_{2}(t)\right|^{3}}\left(\vec{r}_{1}\left(t-\tau_{1,2}(t)\right)-\vec{r}_{2}(t)\right)
\end{gathered}
$$

acts on point $M_{2}$ and the attracting point for point $M_{2}$ at time $t$ is not point $M_{1}$, but point $M_{1}^{*}$, which is determined by vector $\vec{r}_{1}\left(t-\tau_{1,2}(t)\right)$.

The delay of gravity $\tau_{1,2}(t)$ in equality (4) satisfies relation

$$
c \tau_{1,2}(t)=\left|\vec{r}_{1}\left(t-\tau_{1,2}(t)\right)-\vec{r}_{2}(t)\right| .
$$

The existence of functions $\tau_{2,1}(t)$ and $\tau_{1,2}(t)$, which satisfy relations (3) and (5), is shown in [6]. Based on the implicit function theorem [7, p. 449-453], these functions are continuous and differentiable.

Note that the forces $\vec{F}_{2,1, c}(t)$ and $\vec{F}_{1,2, c}(t)$ may differ in magnitude and not be collinear. Also based on (3) and (5) for each point in time $t$

$$
\lim _{c \rightarrow+\infty} \vec{F}_{2,1, c}(t)=\vec{F}_{2,1, \infty}(t)
$$

and

$$
\lim _{c \rightarrow+\infty} \vec{F}_{1,2, c}(t)=\vec{F}_{1,2, \infty}(t) .
$$

Thus, Newton's law of universal gravitation opens the way to a more general law (see (2)-(5)) and coincides with it in the extreme case (with $c=+\infty$ ).

Note that to use formulas (2) and (4), information on vector functions $\vec{r}_{1}(t)$ and $\vec{r}_{2}(t)$ and scalar functions $\tau_{2,1}(t)$ and $\tau_{1,2}(t)$ are needed. These functions can be found using differential equations with a deviating argument, which describe the motion of points $M_{1}$ and $M_{2}$, and are obtained using Newton's second law (see [8]).

\section{The mathematical model of the motion of A FINITE NUMBER OF MATERIAL POINTS}

We pay attention to the mathematical model of the movement of points $M_{i}, i=\overline{1, n}$, with masses $m_{i}, i=\overline{1, n}$, respectively, where $n \geq 2$. The movement of these points will be considered relative to the rectangular coordinate system $x, y, z$ with the origin at some point $O$. We assume that this system is inertial. 


\section{Modeling, control and information technologies - 2020}

The movement of points $M_{i}, i=\overline{1, n}$, can be described by some vector functions $\vec{r}_{i}(t), i=\overline{1, n}$, respectively.

Fix arbitrary points $M_{i}$ and $M_{j}$, where $i, j \in$ $\{1,2, \ldots, n\}$ and $i \neq j$. In accordance with paragraph 2 , point $M_{j}$ attracts point $M_{i}$ with force

$$
\begin{gathered}
\vec{F}_{j, i, c}(t)= \\
=\frac{G m_{j} m_{i}}{\left|\vec{r}_{j}\left(t-\tau_{j, i}(t)\right)-\vec{r}_{i}(t)\right|^{3}}\left(\vec{r}_{j}\left(t-\tau_{j, i}(t)\right)-\vec{r}_{i}(t)\right),
\end{gathered}
$$

where the delay of gravity $\tau_{j, i}(t)$ in equality (6) satisfies relation

$$
c \tau_{j, i}(t)=\left|\vec{r}_{j}\left(t-\tau_{j, i}(t)\right)-\vec{r}_{i}(t)\right| .
$$

We use force $\sum_{j \in N_{i, n}} \vec{F}_{j, i, c}(t)$, which acts on point $M_{i}$ generated by points $M_{j}, j \in N_{i, n}$, where

$$
N_{i, n}=\{1,2, \ldots, n\} \backslash\{i\}, \quad i=\overline{1, n} .
$$

Based on Newton's second law for vector functions $\vec{r}_{i}(t), i=\overline{1, n}$, the relations

$$
m_{i} \frac{d^{2} \vec{r}_{i}(t)}{d t^{2}}=\sum_{j \in N_{i, n}} \vec{F}_{j, i, c}(t), \quad i=\overline{1, n} .
$$

are satisfied. It follows from relations (6) and (7) that vector functions $\vec{r}_{i}(t), i=\overline{1, n}$, are solutions of the following system of differential equations with deviating argument and functional equations

$$
\left\{\begin{array}{l}
\frac{d^{2} \vec{r}_{i}(t)}{d t^{2}}= \\
=\sum_{j \in N_{i, n}} \frac{G m_{j}}{\left|\vec{r}_{j}\left(t-\tau_{j, i}(t)\right)-\vec{r}_{i}(t)\right|^{3}}\left(\vec{r}_{j}\left(t-\tau_{j, i}(t)\right)-\right. \\
\left.-\vec{r}_{i}(t)\right), \quad i=\overline{1, n} \\
c \tau_{j, i}(t)=\left|\vec{r}_{j}\left(t-\tau_{j, i}(t)\right)-\vec{r}_{i}(t)\right| \\
i=\overline{1, n}, \quad j=\overline{1, n}, i \neq j .
\end{array}\right.
$$

Obviously, for each $t$ the correct relations are

$$
\begin{gathered}
\lim _{c \rightarrow+\infty} \tau_{j, i}(t)=0, \quad i=\overline{1, n}, j=\overline{1, n}, i \neq j, \\
\lim _{c \rightarrow+\infty} \vec{F}_{j, i, c}(t)=\vec{F}_{j, i, \infty}(t), \quad i=\overline{1, n}, j=\overline{1, n}, i \neq j,
\end{gathered}
$$

where

$$
\vec{F}_{j, i, \infty}(t)=\frac{G m_{j} m_{i}}{\left|\vec{r}_{j}(t)-\vec{r}_{i}(t)\right|^{3}}\left(\vec{r}_{j}(t)-\vec{r}_{i}(t)\right) .
$$

Therefore, system (8) is a generalization of the classical model of the movement of points $M_{i}, i=\overline{1, n}$,

$$
\left\{\begin{array}{l}
\frac{d^{2} \vec{r}_{i}(t)}{d t^{2}}=\sum_{j \in N_{i, n}} \frac{G m_{j}}{\left|\vec{r}_{j}(t)-\vec{r}_{i}(t)\right|^{3}}\left(\vec{r}_{j}(t)-\vec{r}_{i}(t)\right), \\
i=\overline{1, n},
\end{array}\right.
$$

which is obtained from (8) for $c=\infty$.

When finding the trajectories of movement of points $M_{i}, i=\overline{1, n}$, it is necessary to use, in addition to the equations of system (8), also initial or boundary conditions (see [6], [8], [9]). System (8), together with these conditions, is a mathematical model of the movement of points $M_{i}$, $i=\overline{1, n}$, that takes into account the finiteness of the speed of gravity.

Obviously, system (8), taking into account the initial conditions, can be used as a mathematical model of a stellar system with $n-1$ planets with masses $m_{i}, i=\overline{2, n}$, that move in a force field generated by a star with mass $m_{1}$ and planets.

Note that system (8) contains $n$ vector equations and $n(n-1)$ scalar equations. Therefore, this system is equivalent to a system of scalar equations, the number of which coincides with

$$
3 n+n(n-1)=n^{2}+2 n=(n+1)^{2}-1 .
$$

The classical model (9) of the motion of $n$ material points, which contains $n$ vector equations, is equivalent to a system of $3 n$ scalar equations.

From this and the fact that

$$
\lim _{n \rightarrow \infty} \frac{(n+1)^{2}-1}{3 n}=+\infty,
$$

it follows that even if the complexity of the equations of system (8) is not taken into account, studying system (8) at large $n$ is a more difficult task than studying system (9).

Despite this, it is possible to find such properties of system (8) that are not characteristic of system (9) (see [6], [9]).

\section{The MATHEMATiCAL MODEL OF THE MOVEMENT OF} TWO POINTS

In case $n=2$, the system of equations (8) takes the form

$$
\left\{\begin{array}{l}
\frac{d^{2} \vec{r}_{1}(t)}{d t^{2}}= \\
=\frac{G m_{2}}{\left|\vec{r}_{2}\left(t-\tau_{2,1}(t)\right)-\vec{r}_{1}(t)\right|^{3}}\left(\vec{r}_{2}\left(t-\tau_{2,1}(t)\right)-\right. \\
\left.-\vec{r}_{1}(t)\right), \\
\frac{d^{2} \vec{r}_{2}(t)}{d t^{2}}= \\
=\frac{G m_{1}}{\left|\vec{r}_{1}\left(t-\tau_{1,2}(t)\right)-\vec{r}_{2}(t)\right|^{3}}\left(\vec{r}_{1}\left(t-\tau_{1,2}(t)\right)-\right. \\
\left.-\vec{r}_{2}(t)\right), \\
c \tau_{2,1}(t)=\left|\vec{r}_{2}\left(t-\tau_{2,1}(t)\right)-\vec{r}_{1}(t)\right|, \\
c \tau_{1,2}(t)=\left|\vec{r}_{1}\left(t-\tau_{1,2}(t)\right)-\vec{r}_{2}(t)\right| .
\end{array}\right.
$$

This system, together with the initial or boundary conditions considered in [9], [11] is a mathematical model of 


\section{Modeling, control and information technologies - 2020}

the motion of two points with masses $m_{1}$ and $m_{2}$. System (10) has been thoroughly investigated in the works [8-12].

\section{Additional Remarks AND Literature}

The general case of a system of $n$ bodies, taking into account the finite speed of gravity, was considered by the author in article [6]. This article also pays attention to the construction of a mathematical model of the solar system with a finite speed of gravity and to obtain some new properties for it.

In [9], the problem of two bodies of arbitrary masses was studied taking into account the speed of gravity. The non-Kaplerian behavior and instability of the motion of these bodies are also shown.

In [8], systems of differential equations with delays and constraints on delays and derivatives of solutions, which are used in [9] and [10], are studied.

Articles [13], [14], and [15] show the instability of unbounded trajectories of stellar systems.

\section{REFERENCES}

[1] A. Einstein, On the special and general theory of relativity, Moscow: State Publishing House, 1922.

[2] Yvonne Choquet-Bruhat, General Relativity and Einstein Equations, Oxford University Press, P. 170 (2009).

[3] E. B. Fomalont, S. M. Kopeikin, The Measurement of the Light Deflection from Jupiyer: Experimental Results, Astrophys. J., vol. 598, 704-711 (2003).

[4] B. P. Abbott et al. (LIGO Scientific Collaboration and Virgo Collaboration, Fermi Gamma-ray Burst Monitor, and INTEGRAL), Gravitational Waves and Gamma-Rays from a Binary Neutron Star Merger: GW170817 and GRB 170817A, The Astrophysical Journal, vol. 848, P. L13 (2017). DOI 10.3847/2041-8213/aa920c

[5] V. I. Arnold, V. V. Kozlov, A. N. Neishtadt, "Mathematical aspects of classical and celestial mechanics", Moscow: URSS, 2002.

[6] V. Yu. Slyusarchuk, "Mathematical model of the Solar system with account of gravitation velocity", Nonlinear Oscillations, 2018, vol. 21, № 2, pp. 238-261. DOI 10.1007/s10958-019-04540-2

[7] G. M. Fichtenholtz, "Course in Differential and Integral Calculus", Vol. 1, Moscow: Nauka, 1966.

[8] V. Yu. Slyusarchuk, "Investigation of systems of differential equations with delays and constraints imposed of the delays and derivatives of the solutions", Ukr. Mat. Zh., 2019, vol. 71, № 5, pp. 677-691. DOI 10.1007/s11253019-01673-0

[9] V. Yu. Slyusarchuk, "Non-Keplerian behavior and instability of motion of two bodies caused by finite velocity of gravity", Nonlinear Oscillations, 2018, vol. 21, № 3, pp. 358-419. DOI 10.1007/s10958-019-04550-0
[10] V. Yu. Slyusarchuk, "Kepler's laws and the problem of two bodies with a finite speed of gravity", Bukovinian Mathematical Journal, 2018, vol. 6, № 3-4, pp. 134-151.

[11] V. Yu. Slyusarchuk, "The problem of two bodies with a finite velocity of gravity", The III International Scientific and Practical Conference "Modern Problems of Mathematical Modeling, Automated Control and Information Technologies", November 14-16, 2019, Rivne. Rivne: National University of Water and Enviromental Engineering, 2019, pp. 160-164.

[12] V. Yu. Slyusarchuk, "Application of differential equations with retarded argument and functional equations to the construction of models of stellar systems", Bukovinian Mathematical Journal, 2019, vol. 7, № 2, pp. 105-116.

[13] V. Yu. Slyusarchuk, "Instability of unbounded solutions of evolution equations with operator coefficients commuting with rotation operators", Bukovinian Mathematical Journal, 2019, vol. 7, № 1, pp. 99-113.

[14] V. Yu. Slyusarchuk, "Equations in a Hilbert space whose sets of solutions are invariant with respect to a group isomorphic to a one-parameter group of unitary operators", Ukr. Mat. Zh., 2020, vol. 72, № 1, pp. 86-99.

[15] V. Yu. Slyusarchuk, "Equations whose sets of solutions are invariant with respect to a group of mappings isomorphic to a one-parameter group of rotations", Nonlinear Oscillations, 2020, vol. 23, № 1, pp. 112-123. 